\title{
Regulatory Actions under Adjustment Costs AND THE RESOLUTION OF SCIENTIFIC UNCERTAINTY
}

\author{
David A. Hennessy and GianCarlo Moschini
}

\begin{abstract}
Food system regulators often decide whether to ban existing practices or approve new technologies without conclusive scientific evidence on possible damage and knowing that resolution is likely in the future. In a model with three decision points and stochastic resolution of uncertainty, we study interactions between expected losses due to regulation and information availability when a regulator is deciding on an early reversible ban and on a later reversible ban. Adjustment costs create inertia concerning intermediate signals such that earlier decisions are not overturned, and also a bias against imposing an early ban. The prospect of more later-stage information can increase or decrease the incentive to ban early, but research decreases the incentive to ban early.
\end{abstract}

Key words: Bayesian decision analysis, inertia, precautionary principle, stochastic resolution of uncertainty.

In an increasingly globalized world, regulators in the agricultural and food system are facing a growing demand for action on a number of fronts. Consider, for example, the issues surrounding the diffusion of "genetically modified" (GM) crops - plants transformed by the insertion of foreign genetic material that confers a specific attribute of interest (e.g., herbicide or pest resistance). GM crops have been rapidly adopted by producers in the United States and in a few other countries, but have also generated strong public opposition that is ostensibly rooted in the possibility (as yet unproven) that GM crops pose a risk to human health and the environment.

In Europe, this opposition has resulted in an elaborate regulation-meant to foster food safety, protect the environment, and ensure consumers" "right to know"-that is centered on the notions of labeling and traceability (Commission of the European Communities, 2003). The new framework establishes mandatory labeling for food and feed produced from GM crops, including food from GM products even when it does not contain protein or DNA from the GM crop. A stringent threshold (less that $0.9 \%$ content) is required for food and

\footnotetext{
David A. Hennessy and GianCarlo Moschini are professors at the Department of Economics and affiliates of the Center for Agricultural and Rural Development, Iowa State University. Moschini is also Pioneer Hi-Bred Endowed Chair in Science and Technology Policy. Senior authorship is not assigned.

We thank Bryan Hennessy for comments on the decisionmaking context.
}

feed to avoid the GM label. Such a regulation has so far effectively prevented GM crops from being adopted by E.U. farmers, and it is bound to add considerable cost for suppliers who use GM inputs.

Alternatively, consider the regulatory responses to the discovery of bovine spongiform encephalopathy (BSE), a condition commonly known as "mad cow" disease. BSE first surfaced in the United Kingdom in the 1980s. Because BSE is similar to a sheep disease known as scrapie, it was speculated that scrapie had crossed the species barrier, perhaps through cattle feed containing rendered sheep parts. This led to an early ban of ruminant-derived proteins in ruminant feed, both in the United Kingdom and the European Union. Despite earlier repeated assurances by U.K. authorities that BSE posed no risk of harm for human, public concerns elsewhere led to several bans on importing British bovine products. By the early 1990s, however, BSE cases had been found in several European countries, and concern mounted that in fact BSE could pose a direct risk to humans.

Following additional investigations, the process of mechanically recovering meat was banned in the United Kingdom in 1995 and throughout the European Union in 2000 (Bradley, 2003). In 1996, it was recognized that a lethal new variant on the Creutzfeldt-Jakob disease (CJD) in humans could be related to BSE (Will et al., 1996). As a response to the new information, government blood product 
regulators around the world strengthened prohibitions on the sourcing and uses of human blood. Some of these prohibitions were later relaxed in light of further research (U.S. Food and Drug Administration, 2002).

As firm evidence indicated that species could be silent carriers so that a carrier's body parts could reinfect the primary host (Hill et al., 2000), E.U. regulators extended feed bans and introduced detailed health rules on animal by-products that imposed a large number of use curtailments and documentation requirements (Official Journal of the European Communities, 2002). In 2003, the first two nonimported cases of BSE were detected on the North American continent. Whereas much remains to be understood on BSE and its relationship to CJD (Smith, 2003), Canada and the United States are moving to extend production/processing method and product use proscriptions as well as detection protocols toward those in place in the European Union (Schuff, 2004a). The costs of these prohibitions are large. E.U. member states, for example, collect for disposal 16.24 million tonnes of animal by-products. No longer a revenue source, the materials entails disposal costs of $€ 100$ per tonne (Commission of the European Communities, 2001). In addition, by-product users have had to develop substitutes. The United States produces about 18 million tonnes of affected livestock waste. One estimate of the effects of use bans on animal waste as feed in the United States puts the cost at $\$ 2.17$ billion per annum (Raine, 2003).

Calls for early regulatory action inevitably stumble on a knowledge barrier: rarely is the available scientific information sufficient to support a clear-cut choice when a problem first arises. Whereas an early action has the potential to limit damages when the cause of concern turns out to be correct, regulatory action can also have immediate negative economic impacts on the sector. In such a setting, what attributes should rational regulation display? Some would argue that "If you have to overreact or underreact, it is better to overreact." But imperfect scientific knowledge implies that trade-offs between potential benefits and lost economic opportunities are inherent in any meaningful regulatory choice. Perhaps more important, scientific knowledge expands over time so that uncertainty about

\footnotetext{
${ }^{1}$ Representative Gil Gutknecht stated that on January 21, 2004, at a U.S. House of Representatives Committee on Agriculture hearing on new regulations to control BSE (Schuff, 2004b).
}

the questions facing regulators is typically resolved over time. The prospect of eventual resolution ought to be accounted for when taking the initial regulatory action.

In this article, we explicitly address the issue of how the evolution of scientific knowledge may be expected to affect the choices of a rational, risk-neutral regulator. The calculus of the problem faced by regulators of course includes many facets. Foremost is the mandate to prevent negative effects on public health and the environment. But regulators must also consider the economic consequences of their choices, and affected industries are usually active in representing the costs that may result from various regulatory alternatives. We focus thus on the effects of the "adjustment costs" that are implied by a new regulation, and on how these costs interact with a fundamental feature of the regulators' decision problem. This feature is that scientific knowledge on an issue is typically imperfect, and is likely to improve over time. Aggressive regulatory activism may prevent problems from escalating and translating into harm to the public. But because future knowledge is always expected to be better than current knowledge, future actions may be more effective than current actions. Thus, the possibility of future regulatory actions, to be based on information presently unavailable, necessarily affects the optimality of regulatory choices.

The model we develop can be summarized as follows. At time $t=0$ a new regulation is considered as a response to emerging concerns (e.g., banning certain feeding practices that might be linked to the onset of BSE in cattle). An action at this time can produce benefits, the exact magnitude of which is unknown. But a new regulatory action also entails two distinct costs: an opportunity cost because alternative practices are more expensive, and also an adjustment cost. At some (unknown) future date $t=\kappa_{1}$, more information becomes available and the regulator may change the action chosen at date $t=0$. A new action at this point, or a reversal of the early action, also incurs adjustment costs. Eventually, after an additional uncertain time interval, at date $t=\kappa_{1}+\kappa_{2}$, full information becomes available and the regulator's decision can be revisited for the final time. In this setting, we show that adjustment costs and the resolution of uncertainty play an important and interactive role in affecting the $t=$ 0 decision. We also study some effects due to the expected gradual resolution of uncertainty, both when expected resolution is exogenous 
and when research funding can advance the arrival of new information.

Whereas we explore a new modeling approach by representing the resolution of uncertainty in terms of a stochastic arrival of new information, the economics of our study is rooted in an established strand of literature originated with Arrow and Fisher (1974), and Henry (1974), who modeled the effect of the availability of subsequent information on an irreversible decision. In their studies, the availability of later information encourages the early-stage preservation of options over the set of actions that could be made after the laterstage information does become available. This creates the so-called quasi-option value, which can play an important role in cost-benefit analyses of environmental problems (Hanemann, 1989). ${ }^{2}$

Kolstad (1996a) has identified a second avenue for expected future knowledge to affect the early-stage decision calculus: when capital dedicated to solving a potential problem is sunk and irretrievable. Sunk costs generate an incentive to act later, after better information has become available, so as to avoid a possible waste. This is a form of real option to defer investment, as in Dixit and Pindyck (1994). Thus, Kolstad's (1996a) model identifies a tension between two types of irreversibilitiesthe prospect of irreversible damage favors earlier preventative action, but the need for irreversible investments to support current action favors later actions. In our context, a regulator's decisions are assumed to be completely reversible, so that the first kind of irreversibility (the contraction in the future action space that featured prominently in Arrow and Fisher (1974)) is not an issue. On the other hand, the irreversibility in our model is due to the fact that new regulation involves sunk adjustment costs (because of the possible need for new dedicated capital, and because the changes incurred by the production system may involve loss of rents on existing assets, search costs, management time, and loss of markets). In addition, as will be explained, adjustment costs introduce nonconvexities that affect the role of subsequent information. This issue does not appear to have been studied elsewhere.

The real option models rooted in Dixit and Pindyck, such as Pindyck's (2002) study of pol-

\footnotetext{
${ }^{2}$ These original insights have been developed and applied in several environmental economics studies, particularly with reference to the problem of "global warming" (e.g., Kolstad, 1996b; Ulph and Ulph, 1997; Fisher and Narain, 2003).
}

icy adoption timing, are also germane to our work. But note that these studies concern the effects of uncertainty on initial decisions, and not necessarily the structure of sequential revelations of information that is the focus of our article. Indeed, such real option models generally do not distinguish between the stock of information that has yet to be revealed (i.e., the amount of prior uncertainty) and the rate of flow of new information, so that an increase in the flow of new information at a time point is confounded with an increase in the amount of prior uncertainty. Our model will fix the amount of prior uncertainty and change the time path of information flow. Jones and Ostroy (1984) is the study closest to ours. There the interest is in how flexibility, as represented by low switching costs, interacts with quality of information in determining the ex ante value of an objective function to be optimized. They seek to identify economic motives for taking flexible positions. In contrast, our interest is in understanding the determinants of a regulator's actions.

Related literature also includes Gollier, Jullien, and Treich (2000), who introduce a risk aversion motive for precautionary behavior in the face of scientific uncertainty. The objective there is to provide an economic rationalization for the so-called precautionary principle, a concept being increasingly advocated to deal with environmental and other risks. ${ }^{3}$ In that model, the tension between the "now" and "wait" motives for action emerges from subtle risk management and wealth effects. ${ }^{4}$ Whereas our approach could be formulated to account for risk aversion with wealth as well, here we abstract from such considerations, and the effects that we uncover apply even under risk neutrality.

\footnotetext{
${ }^{3}$ The principle of precautionary action was adopted in the 1992 United Nations Conference on Environment and Development, where it was explicitly embedded in the Rio Declaration as follows: "Lack of full scientific certainty shall not be used as a reason for postponing cost-effective measures to prevent environmental degradation" (Freestone and Hey, 1996). The original intent to apply the precautionary approach to environmental risks has recently been expanded by the European Union to include food safety as well (Commission of the European Communities, 2000). There is an ongoing debate as to the proper economic interpretation of the precautionary principle, but a cogent interpretation in terms of traditional cost-benefit analysis under risk is possible (Gollier, 2001).

${ }^{4}$ Another article that deals with how risk aversion interacts with the timing of information availability is Eeckhoudt, Gollier, and Treich (2005). In a three-date model, they show that earlier resolution of uncertainty about future income should reduce the incentive to make precautionary savings when the same utility function applies across periods and has a positive third derivative.
} 
In what follows we develop our model within a formal Bayesian decision framework. We first analyze a two-date version of the model when the probabilistic resolution of uncertainty is held as exogenous. A choice may be taken at time $t=0$, or it may be postponed until a later date when uncertainty is resolved. This basic setting, with no intermediate revelation of information and/or flexibility, allows a clear appreciation of the various elements of the model and provides an important benchmark for later analysis. We then consider a threedate, two-period model where uncertainty is resolved gradually and where there is intermediate flexibility-i.e., the regulator can adjust its choice to the attainment of partial new knowledge. Among other results, the model shows that the extent of investment in research on the problem decreases the incentive to ban early. Our final analysis section returns to the two-date version of the model, but endogenizes the resolution of uncertainty by optimizing over research expenditures.

\section{Baseline Model: No Intermediate Information and/or Flexibility}

The prototypical regulator problem we envision in our model is as follows. There is growing concern that an existing practice, or emerging new technology, may pose the threat of some harm to humans and/or the environment. To root our model in an explicit Bayesian framework, we postulate that there are two states of the world: it is true that the practice of interest causes harm $(T)$ and it is false that the practice in question causes harm $(F)$. At time $t=0$, there is a probability $q$ that the state of the world is $T$ and probability $1-q$ that the state of the world is $F$. For concreteness we suppose that the regulator's problem concerns an existing practice, and that the choice is whether to ban the existing practice or to defer such a choice to the future. Thus, in the model there are two actions, to ban $(B)$ and to not ban $(N)$. Uncertainty will be resolved at some future date $\kappa>0$, when future knowledge will provide perfect information on the true state of the world. This information is free to the regulator, and the arrival date is considered for now to be exogenous to the regulator. At time $t=0$, the arrival date $\kappa$ is a random variable.

As mentioned earlier, we postulate two distinct costs that result from a regulatory action. First, there is a cost to banning a technology because it forces producers to rely on less ef- ficient alternatives. We represent that by the instantaneous cost $C$ (thus, $C$ is the parameter of a constant flow of costs to be incurred as long as the regulation is in place). In addition, we allow for adjustment (switching) costs to be incurred when the system first adjusts to a new regulatory regime. Specifically, when the new regulation is first imposed (regardless of whether it is now or in the future) a one-time adjustment cost of $\delta_{1}>0$ is to be incurred. Furthermore, if a new regulation is reversed in the future, the cost $C$ ceases to apply (producers can revert to using the most efficient technology) but a new adjustment cost $\delta_{2}>0$ is necessary to transition back to a new equilibrium. ${ }^{5}$

On the benefits side, we postulate that there is an (instantaneous) benefit of $D>0$ that arises from banning a harmful practice (thus, $D$ can be interpreted as the damage that arises if true state is $T){ }^{6}$ With $r$ as the continuoustime discount rate, we shall assume that $D>$ $C+\delta_{1} r$. This ensures that a regulatory action would be prima facie desirable if it were known with certainty that the practice in question is indeed harmful. Were this condition not valid then the rent on the lump sum adjustment cost, $\delta_{1} r$, together with the flow cost, $C$, would outweigh damage done and a ban would never be optimal. $^{7}$

The payoff to the regulator is the total (expected) cost (including possible damages, technology replacement, and adjustment costs). The realized cost is written as $R(a, s, \kappa)$, where $a \in\{B, N\}$ is the action chosen, $s \in\{T, F\}$ is the realized state of the world, and $\kappa \in(0, \infty)$ is the time at which uncertainty is resolved. The regulator's decision problem is given in figure 1 , where payoffs are evaluated at $t=$ 0 present values. In this representation, $\kappa$ is a random variable with distribution $f(\kappa)$. While

\footnotetext{
5 These adjustment costs may also be time-dependent. Suppose regulators do not ban promptly a practice after the public becomes aware of a concern with it, but do ban after additional information becomes available. Then consumers may perceive that the process does not work well to safeguard public well-being and avoid the good even after the practice has been banned. A large market disruption may follow, which can be viewed as an adjustment costs $\delta_{1}$ that is time dependent. For tractability, we ignore this issue in our model.

${ }^{6}$ While the magnitude of the suspected damage is known, a probability can be assigned to the event that this damage is occurring over time. In reality, the extent of damage is likely to follow a continuous distribution. In addition, knowledge on the extent of damage and the probability that any damage is occurring at all will accrete incrementally. Our model simplifies such matters in order to obtain clearer insights.

${ }^{7}$ In reality, the regulator has available a suite of alternative actions with distinct consequences for the magnitudes of $D$ and $C$. We focus on a single extreme choice because we do not believe that allowing for an arbitrary array of choices would provide additional insights.
} 


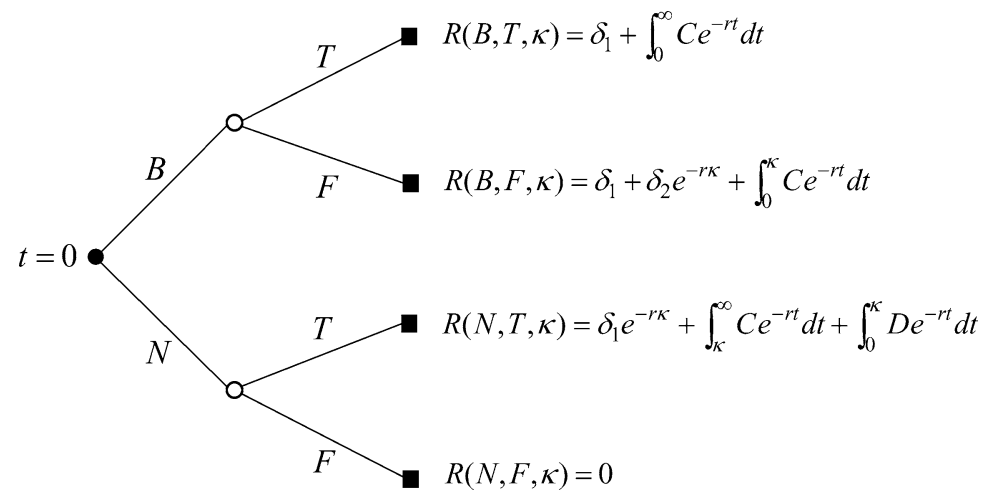

Figure 1. Regulator payoffs with no intermediate information and/or flexibility

our model has $\kappa$ as random, we could readily adapt it so that $C$ or $D$ were random. The action-conditioned expected losses to the regulator of a given initial action, written as $V(a)$, are

$$
\begin{aligned}
V(B)= & \int_{0}^{\infty}[q R(B, T, \kappa) \\
& +(1-q) R(B, F, \kappa)] f(\kappa) d \kappa \\
V(N)= & \int_{0}^{\infty}[q R(N, T, \kappa) \\
& +(1-q) R(N, F, \kappa)] f(\kappa) d \kappa .
\end{aligned}
$$

Equation (1) represents expected losses (including switching costs) from accepting the truth of the hypothesis that the practice causes harm when in fact it does not, i.e., statistical type II error. Equation (2) represents expected losses from rejecting the hypothesis when in fact harm is caused, i.e., type I error.

To gain insights into the problem, we shall assume that $\kappa$ is exponentially distributed such that the density function is $f(\mathrm{\kappa})=h e^{-h \kappa}$ (Taylor and Karlin, 1984). ${ }^{8}$ Parameter $h$ represents the "hazard rate," which is a constant for the memoryless exponential distribution. Note that this parameter is inversely related to the expected date of arrival of knowledge, specifically $E[\kappa]=1 / h$. Given this parameterization, we obtain

\footnotetext{
${ }^{8}$ The exponential distribution is widely used in the R\&D literature to model innovation arrival, at least since Loury (1979). Its main merit is that it is memoryless so that expected time to arrival of information is independent of how long the scientific uncertainty has persisted. In addition to being convenient, this property is also very appealing in our context because it is "neutral" with respect to the structure of arrival of information.
}

$$
\begin{aligned}
V(B)= & \delta_{1}+q\left(\frac{C}{r}\right) \\
& +(1-q)\left(\frac{C+\delta_{2} h}{r+h}\right) \\
V(N)= & q\left(\frac{C}{r}+\frac{D-C}{r+h}+\frac{\delta_{1} h}{r+h}\right) .
\end{aligned}
$$

In this setting, a ban is optimal if it leads to a lower total cost, i.e., if $V(B)<V(N)$, which requires

$$
q>\frac{C+\delta_{1} r+\left(\delta_{1}+\delta_{2}\right) h}{D+\left(\delta_{1}+\delta_{2}\right) h} \equiv q_{\delta, h} .
$$

Relation (5) allows for several conclusions. Consider first the special case of no adjustment costs (i.e., $\delta_{1}=\delta_{2}=0$ ). In such a case, a ban is warranted if $q>C / D \equiv q_{0}$ (and no ban otherwise); that is, the standard cost-benefit criterion applies. Next, consider the effect of positive adjustment costs $\delta_{i}>0, i \in\{1,2\}$, but assume that there is no expected resolution of uncertainty (meaning that $h=0$ so that expected time to resolution is infinite). In such a case, a ban is warranted if $q>\left(C+\delta_{1} r\right) / D \equiv$ $q_{\delta}>q_{0}$. Thus, the existence of adjustment costs tilts the optimal decision toward the status quo and the motive is to avoid incurring adjustment costs.

Finally, with positive adjustment costs as well as expected resolution of uncertainty (i.e., $h>0$ ), a ban is warranted if $q>q_{\delta, h}$, where $q_{\delta, h}>q_{\delta}$ is due to (5). Thus, given adjustment costs, the bias in favor of the status quo is strengthened for $h>0$ when compared with $h=0$. Also, as the expected resolution of uncertainty moves closer in time (i.e., $h$ gets larger), the threshold level $q_{\delta, h}$ increases so that 
waiting becomes more and more attractive. The marginal impact of the adjustment cost parameters is also easily illustrated. Specifically, $\partial q_{\delta, h} / \partial \delta_{1}>\partial q_{\delta, h} / \partial \delta_{2}>0$. Thus, increases in the magnitude of adjustment costs increase the hurdle to be met to justify action at $t=$ 0 , with the highest effect belonging to the adjustment cost incurred with the first regulation $\left(\delta_{1}\right)$. We can summarize the foregoing as follows.

RESULT 1. The existence of adjustment costs biases the regulator's optimal choice towards the status quo. The existence of adjustment costs also creates scope for the resolution of uncertainty to impact optimal decisions. As the expected resolution of uncertainty moves closer in time, the status quo looks more and more attractive.

In what follows, we extend the model to allow for the adaptation in regulators' decisions that improved (but not perfect) intermediate information makes possible.

\section{Gradual Resolution of Uncertainty: A Three-Date, Two-Period Model}

Truth in science seldom dawns with a single discovery, but rather it is established after a sequence of discoveries. To capture this feature of the real world in an effective manner, we postulate that uncertainty is resolved in two stages (e.g., two discoveries are required to ascertain the truth, and these discoveries are necessarily sequential). At time $t=0$, there is a probability $q$ that the true state of the world is $T$, and probability $1-q$ that the true state is $F$. At some future date $t=\kappa_{1}>0$, the probability $q$ is updated, say, to $\tilde{q}$, and the initial decision can be changed at this future date. ${ }^{9}$ The possibility of revising the decision after new information becomes available should mean that the decision-maker is interested in how the signals are distributed because the revised action will depend on the new information.

At $t=0$, the distribution of signal $\tilde{q}$ is $G(\tilde{q})$ : $[0,1] \rightarrow[0,1]$ where set probability measures

\footnotetext{
${ }^{9}$ For example, $q=0.5$ while the updated probability is distributed uniformly on $\tilde{q} \in[0,1]$. The probabilities become more dispersed after the new information becomes available. A very dispersed distribution on $\tilde{q}$ reflects a very informative intermediate signal. Two extremes are when $\tilde{q}=q$ (so that no new information emerges at time $\kappa_{1}$ ), and when $\tilde{q}$ only has values 0 or 1 (so that the $t=\kappa_{1}$ signal is fully informative). The latter distribution is clearly more dispersed than the former.
}

are represented as $G(S), S \subseteq[0,1]$. We assume that there are no atoms, i.e., no points of discontinuity on $G(\tilde{q}){ }^{10}$ To ensure internal consistency, it must be that $q=\int_{0}^{1} \tilde{q} d G(\tilde{q})$. Collectively, the $t=0$ information structure is described as $\{\tilde{q} ; G(\tilde{q})\}$. At some further point in time $t=\kappa_{1}+\kappa_{2}>\kappa_{1}$, further knowledge will reveal the true state of the world, and the final (ex post optimal) decision is implemented. Regulation $a_{0} \in\left\{B_{0}, N_{0}\right\}$ can be taken at $t=$ 0 , and regulation $a_{1} \in\left\{B_{1}, N_{1}\right\}$ can be taken at $t=\kappa_{1}$ (although the action set is really the same at the two dates, for notational clarity we carry the subscript for $B$ and $N$ as well). ${ }^{11}$ The problem facing the regulator, as a game against nature, is illustrated in figure 2 , where each terminal node represents the discounted (at $t=0)$ state contingent loss. Note that, to facilitate tractability, from now onward we assume that $\delta_{1}=\delta_{2} \equiv \delta$ (i.e., the same adjustment cost applies each time a regulatory regimes is changed). ${ }^{12}$

To proceed, as in the earlier section, we assume that $\kappa_{1}$ is exponentially distributed with density $f\left(\kappa_{1}\right)=h_{1} e^{-h_{1} \kappa_{1}}$, where $h_{1}$ is the hazard rate for the first resolution of uncertainty (e.g., Taylor and Karlin). Once $\kappa_{1}$ is realized, the second discovery at $\kappa_{2}$ becomes possible and $\kappa_{2}$ is also distributed exponentially, with density function $f\left(\kappa_{2} \mid \kappa_{1}\right)=h_{2} e^{-h_{2} \kappa_{2}}$, where $h_{2}$ is the hazard rate for the second resolution of uncertainty. Given these stochastic assumptions, we can take expectations over the distribution of random dates $\left(\kappa_{1}, \kappa_{2}\right)$ and the distribution of the states of the world. The decision problem of the regulator can thus be illustrated as in figure 3 , where $V\left(a_{0}, a_{1} \mid \tilde{q}\right)$ denotes the signal-conditioned, discounted expected costs (with $a_{0}$ denoting the action taken at $t=0$ and $a_{1}$ denoting the action taken at time $t=\kappa_{1}$ ).

Evaluating the terms in the terminal nodes of figure 2 (by using the exponential distributions for $\kappa_{1}$ and $\kappa_{2}$ ) and taking expectations over the states of the world, the

\footnotetext{
10 This means that allocation of boundary points on interval subsets of $[0,1]$ are of no consequence.

11 The capacity to adapt to decisions matters and should alter preferences over information (Mossin, 1969; Spence and Zeckhauser, 1972; Kreps and Porteus, 1978). Our interest is not in preferences over information structures, but rather in how information structure and the temporal structure of later decisions affect earlier actions taken in the presence of adjustment costs.

${ }^{12}$ Allowing $\delta_{1} \neq \delta_{2}$ has no qualitative effect on the results.
} 


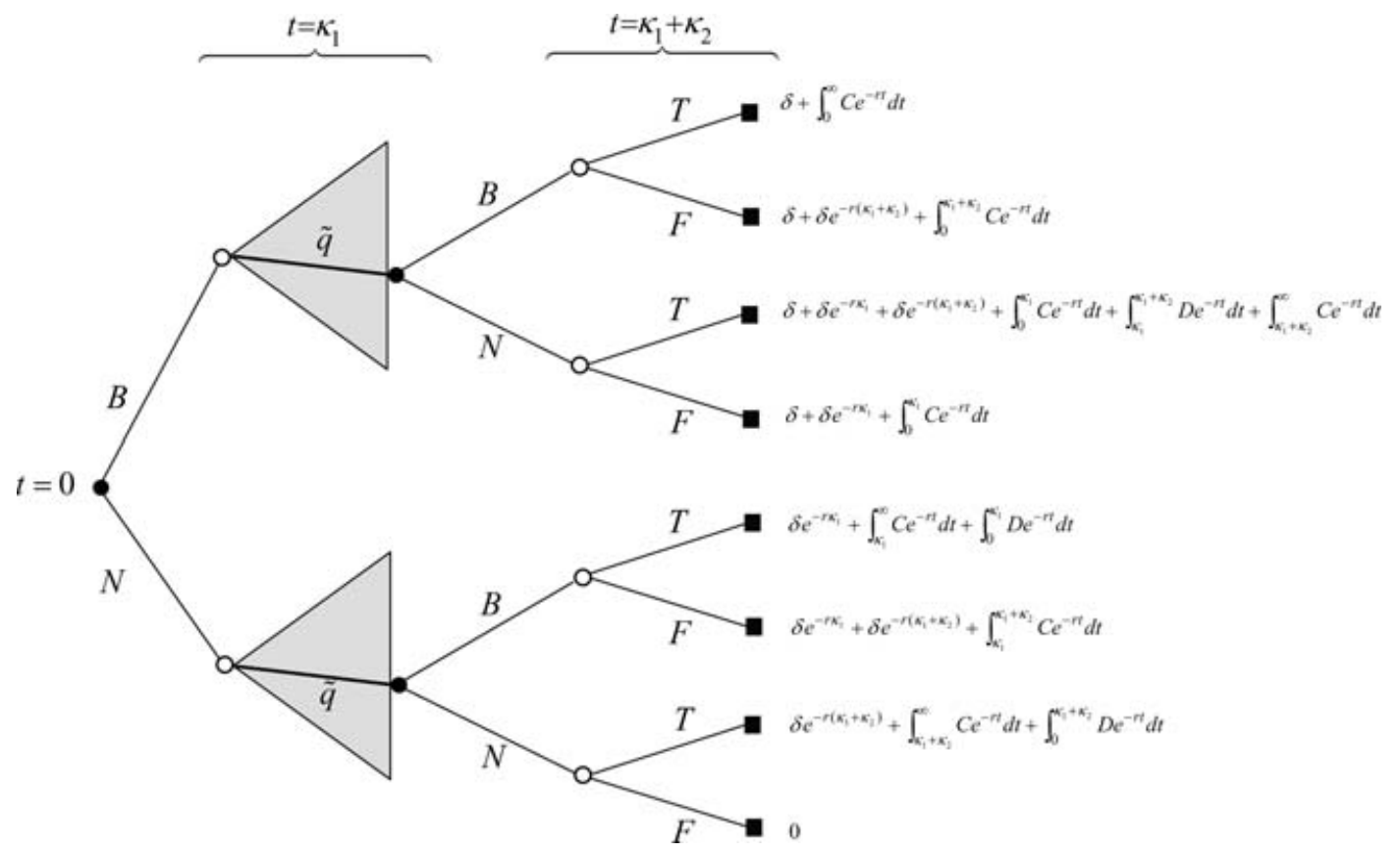

Figure 2. Three-date, two-period model structure, with discounted state-contingent loss

signal-conditioned expected regulation costs are computed as follows:

(6) $V\left(B_{0}, N_{1} \mid \tilde{q}\right)=\delta+\frac{C+\delta h_{1}}{r+h_{1}}$

$$
+\tilde{q} \frac{h_{1}\left[D r+h_{2}(C+\delta r)\right]}{r\left(r+h_{1}\right)\left(r+h_{2}\right)}
$$

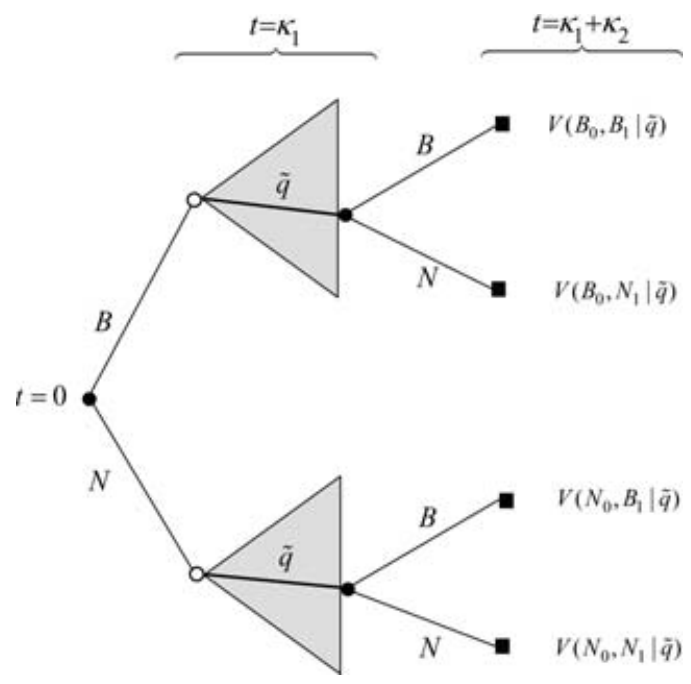

Figure 3. Regulator payoffs with intermediate information and flexibility
(7)

$$
\begin{aligned}
V\left(B_{0}, B_{1} \mid \tilde{q}\right) & \\
= & \delta+\frac{C\left(r+h_{1}+h_{2}\right)+\delta h_{1} h_{2}}{\left(r+h_{1}\right)\left(r+h_{2}\right)} \\
& +\tilde{q} \frac{h_{1} h_{2}(C-\delta r)}{r\left(r+h_{1}\right)\left(r+h_{2}\right)}
\end{aligned}
$$

(8) $\quad V\left(N_{0}, N_{1} \mid \tilde{q}\right)$

(9) $\quad V\left(N_{0}, B_{1} \mid \tilde{q}\right)$

$$
=\tilde{q} \frac{h_{1} h_{2}(C+\delta r)+\operatorname{Dr}\left(r+h_{1}+h_{2}\right)}{r\left(r+h_{1}\right)\left(r+h_{2}\right)}
$$

$$
=\frac{\delta h_{1}}{r+h_{1}}+\frac{h_{1}\left(C+\delta h_{2}\right)}{\left(r+h_{1}\right)\left(r+h_{2}\right)}
$$

$$
+\tilde{q} \frac{\operatorname{Dr}\left(r+h_{2}\right)+h_{1} h_{2}(C-\delta r)}{r\left(r+h_{1}\right)\left(r+h_{2}\right)} .
$$

The linearity of expected costs in $\tilde{q}$ will prove to be useful when identifying the regulator's optimal $t=0$ decisions.

Optimal Initial Decision with No Flexibility

In order to benchmark the three-date, twoperiod model in (6)-(9) relative to the baseline model in (3)-(4) we represent the case of no flexibility as the constraint $a_{0}=a_{1}$. Thus, only the payoffs $V\left(N_{0}, N_{1} \mid \tilde{q}\right)$ and $V\left(B_{0}, B_{1} \mid \tilde{q}\right)$ 
can be realized. For a ban at $t=0$ to be optimal, it is necessary that $E\left[V\left(N_{0}, N_{1} \mid \tilde{q}\right)\right]>$ $E\left[V\left(B_{0}, B_{1} \mid \tilde{q}\right)\right]$, which requires: ${ }^{13}$

$$
q>\frac{\left(r+h_{1}+h_{2}\right)(C+\delta r)+2 \delta h_{1} h_{2}}{\left(r+h_{1}+h_{2}\right) D+2 \delta h_{1} h_{2}} \equiv \hat{q} .
$$

Notice that $\hat{q}<1$ iff $C+\delta r<D$. Henceforth, and for the reason given in the baseline model, we make the assumption that $C+\delta r<$ $D$. Then $d \hat{q} / d \delta>0$ so that the interval in which a ban occurs, $(\hat{q}, 1]$, contracts as the magnitude of adjustment costs increases. The same is true of an increase in either $h_{1}$ or $h_{2}$. Essentially, therefore, this case of no flexibility conforms to the earlier model and Result $1 .{ }^{14}$ As Ostroy and Jones have pointed out in their Proposition 2, when there is no capacity to respond to new information then optimal initial decisions depend only on the initial knowledge, i.e., on $q$.

\section{Flexible Environment-Optimal Intermediate Decisions}

To analyze the optimal intermediate decisions under flexibility, it is convenient to rewrite the signal-conditioned expected costs as

$$
\begin{aligned}
V\left(B_{0}, B_{1} \mid \tilde{q}\right)= & V\left(B_{0}, N_{1} \mid \tilde{q}\right) \\
& +\left(q^{-}-\tilde{q}\right) K \\
V\left(N_{0}, B_{1} \mid \tilde{q}\right)= & V\left(N_{0}, N_{1} \mid \tilde{q}\right) \\
& +\left(q^{+}-\tilde{q}\right) K
\end{aligned}
$$

where $^{15}$

$$
\begin{aligned}
& q^{-} \equiv \frac{C-\delta r}{D+2 \delta h_{2}} \\
& q^{+} \equiv \frac{C+\delta r+2 \delta h_{2}}{D+2 \delta h_{2}} \\
& K \equiv \frac{h_{1}\left(D+2 \delta h_{2}\right)}{\left(r+h_{1}\right)\left(r+h_{2}\right)}
\end{aligned}
$$

Thus, if the initial decision were $B_{0}$, then the regulator should ban at $t=\kappa_{1}$ when-

\footnotetext{
${ }^{13}$ Note that this expectation operator is defined over the distribution of $\tilde{q}$, and it is evaluated at date $t=0$.

${ }^{14}$ Equations (5) and (10) are obviously similar, with the differences arising because $\delta_{1}=\delta_{2}=\delta$, because we have broken the period of uncertainty resolution into two segments, and because the sum $\left(\kappa_{1}+\kappa_{2}\right)$ does not quite have an exponential distribution if $\kappa_{1}$ and $\kappa_{2}$ possess the assumed exponential distribution.

15 A quantity very similar to $q^{+}$was encountered in relation (5) above.
}

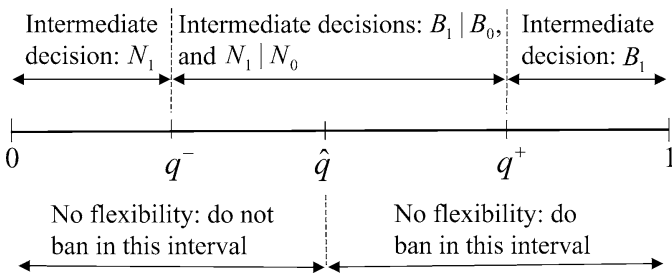

Figure 4. Adjustment cost induced inertia in regulation at a later date

ever $V\left(B_{0}, N_{1} \mid \tilde{q}\right)>V\left(B_{0}, B_{1} \mid \tilde{q}\right)$, i.e., whenever $\tilde{q}>q^{-}$. On the other hand, if the initial decision were $N_{0}$, then the regulator should impose a ban at $t=\kappa_{1}$ whenever $\tilde{q}>q^{+}$.

It is readily shown that $q^{-} \leq \hat{q} \leq q^{+}$, where $\hat{q}$ is defined in equation (10). To see this, write $\hat{q}=\left[C+\delta r+2 \gamma \delta h_{2}\right] /\left[D+2 \gamma \delta h_{2}\right]$ where $\gamma \equiv$ $h_{1} /\left(r+h_{1}+h_{2}\right)$. Because $\gamma \in[0,1]$, it is clear that $q^{-} \leq \hat{q}$. Also, $d \hat{q} / d \gamma \geq 0$, and $\hat{q}=q^{+}$iff $\gamma=1$, so we can conclude that $\hat{q} \in\left[q^{-}, q^{+}\right]$. The role of flexibility on intermediate-stage decisions is summarized in Result 2 and depicted in figure 4.

RESUlt 2. Optimal intermediate decisions, given flexibility and under adjustment costs, display some inertia. Specifically, there is an interval $\left[q^{-}, q^{+}\right]$such that when the signal falls in this range the optimal intermediate action is to carry on with the initial action, regardless of which initial action was chosen.

Thus, Result 2 uncovers a clear example of what Dixit and Pindyck call "hysteresis," specifically an instance of path dependency in (optimal) economic decisions. Since $d q^{+} / d \delta>$ $0>d q^{-} / d \delta$, the interval expands with the extent of adjustment costs. If, for example, a government chooses to subsidize adjustment by partial payment of costs incurred, then this band would contract. In our setting, though, there is no reason to support such an intervention because the inertia is optimal. The subsidy would transfer incidence of costs and also encourage excessive adjustment. In what follows we address the question of how such hysteresis affects the initial decision.

\section{Flexible Environment-Optimal Initial Decisions}

Having characterized the (constrained) optimality of subsequent decisions, we can now consider how optimal initial decisions are taken in a context where it is known that some information will be revealed (i.e., some uncertainty will be resolved) and that the initial 
decision can be revisited when that happens (i.e., there is flexibility). Let $a_{1}^{*} \in\left\{B_{1}, N_{1}\right\}$ denote the optimal intermediate decision, as discussed in the preceding section. For signal action $a_{0} \in\left\{N_{0}, B_{0}\right\}$, the regulator's expected losses (conditional on $\tilde{q}$ ) are

$$
\begin{aligned}
V( & \left.N_{0}, a_{1}^{*} \mid \tilde{q}\right) \\
= & \min \left\{V\left(N_{0}, N_{1} \mid \tilde{q}\right), V\left(N_{0}, B_{1} \mid \tilde{q}\right)\right\} \\
= & \left(\frac{D}{r+h_{1}}+\frac{h_{1} h_{2}(C-\delta r)}{r\left(r+h_{1}\right)\left(r+h_{2}\right)}\right) \tilde{q} \\
\quad & \quad K \min \left[\tilde{q}, q^{+}\right]
\end{aligned}
$$

$$
\begin{aligned}
V\left(B_{0}, a_{1}^{*} \mid \tilde{q}\right) \\
=\min \left\{V\left(B_{0}, N_{1} \mid \tilde{q}\right), V\left(B_{0}, B_{1} \mid \tilde{q}\right)\right\} \\
=\frac{C+\delta r+2 \delta h_{1}}{r+h_{1}}+\frac{h_{1} h_{2}(C-\delta r)}{r\left(r+h_{1}\right)\left(r+h_{2}\right)} \tilde{q} \\
\quad+K \min \left[\tilde{q}, q^{-}\right] .
\end{aligned}
$$

To determine which of the two initial actions is optimal at $t=0$ it is necessary to integrate over the distribution of the signal $\tilde{q}$. Thus, the optimal decision at $t=0$ will revolve around what we shall call the "policy function," $\Delta \equiv E\left[V\left(B_{0}, a_{1}^{*} \mid \tilde{q}\right)\right]-E\left[V\left(N_{0}, a_{1}^{*} \mid \tilde{q}\right)\right]$. If $\Delta<0$, then it is optimal to ban at $t=0$, whereas if $\Delta \geq 0$, then it is optimal to not ban at $t=0$. By using equations (16) and (17), the policy function can be expressed as

$$
\begin{aligned}
\Delta= & \frac{C+\delta r+2 \delta h_{1}-q D}{r+h_{1}} \\
& +E\left[A\left(\tilde{q} ; q^{+}, q^{-}\right)\right]
\end{aligned}
$$

where $q=E[\tilde{q}]$, as noted earlier, and

$$
\begin{aligned}
& A\left(\tilde{q} ; q^{+}, q^{-}\right) \\
& \quad \equiv K\left(\min \left[\tilde{q}, q^{-}\right]-\min \left[\tilde{q}, q^{+}\right]\right) .
\end{aligned}
$$

The function $A\left(\tilde{q} ; q^{+}, q^{-}\right)$plays an important role in our analysis, and it is depicted in figure $5 .{ }^{16}$ It is bounded on $\left[-2 \delta h_{1} /\left(r+h_{1}\right), 0\right]$ so that $\Delta \geq(C+\delta r-q D) /\left(r+h_{1}\right) \geq(C-q D) /$ $\left(r+h_{1}\right)=\left.\Delta\right|_{\delta=0}$. This means that the existence of adjustment cost $\delta$ introduces a predisposition to not ban. Similarly, $\Delta \leq(C+\delta r+$

\footnotetext{
${ }^{16}$ With $\delta_{1} \neq \delta_{2}$ figure 5 would modify slightly. But the critical attributes of concave over $\left[0, q^{+}\right)$and convex over $\left(q^{-}, 1\right]$ continue to apply.
}

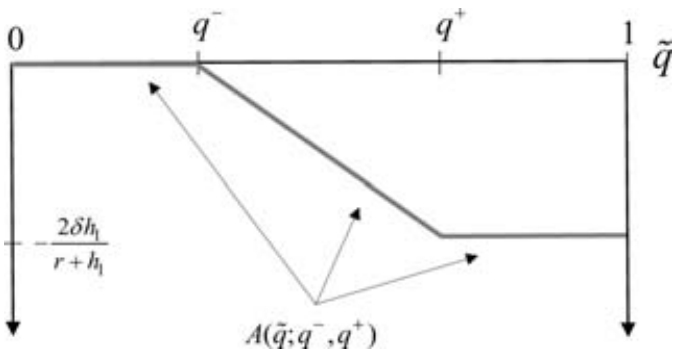

Figure 5. $A\left(\tilde{q} ; q^{-}, q^{+}\right)$as signal value changes

$\left.2 \delta h_{1}-q D\right) /\left(r+h_{1}\right)=\left.\Delta\right|_{\delta=0}+\delta\left(r+2 h_{1}\right) /$ $\left(r+h_{1}\right)$.

We can summarize the foregoing as follows.

RESULT 3. In the presence of adjustment costs, the optimal decision is to not ban if $(C+\delta r) /$ $D \geq q$ and it is to ban if $\left(C+\delta r+2 \delta h_{1}\right) / D<$ $q$. In these cases, the informativeness of the $t=$ $\kappa_{1}$ information structure $\{\tilde{q} ; G(\tilde{q})\}$ has no effect on the $t=0$ decision. In addition, if it is optimal to ban in the presence of adjustment costs then it is optimal to ban in the absence of adjustment costs, i.e., $\Delta \geq\left.\Delta\right|_{\delta=0}$ so that $\Delta<0$ implies $\left.\Delta\right|_{\delta=0}$ $<0$.

Before inquiring into how quality of information matters in the presence of adjustment costs, we remark that it does not matter when $\delta=0$. From $\left.\Delta\right|_{\delta=0}=(C-q D) /\left(r+h_{1}\right)$, it is clear that whether ban or not ban at $t=0$ supports lower $t=0$ expected loss under $\delta=$ 0 depends only on the ex ante signal mean $\mathrm{q}$ and not on any other attributes of $\{\tilde{q} ; G(\tilde{q})\}$. If $q>C / D$, then the $t=0$ decision is to ban. If $q \leq C / D$, then the $t=0$ decision is to allow the practice. Only $q$ matters in determining the $t=0$ decision under $\delta=0$ because the $t=\kappa_{1}$ expected loss is invariant to the $t=0 \mathrm{de}$ cision. Better information will allow for betterquality $t=\kappa_{1}$ decisions, thus decreasing ex ante expected loss, but the $t=\kappa_{1}$ state-conditional decisions are separated from the $t=0$ decision. The separation fails when adjustment costs are incurred. We turn now to the interval of ex ante probability not covered in Result 3, that is, $q \in$ $\left((C+\delta r) / D,\left(C+\delta r+2 \delta h_{1}\right) / D\right]$.

\section{Effects of the "Quality" of Information}

To frame the issue it is useful to consider the (degenerate) polar case that arises when there is no meaningful expected resolution of uncertainty. Specifically, if it were known that at $t=$ $\kappa_{1}$ the probability of the practice in question causing harm is unchanged-that is, $\tilde{q}=q$ for 
all possible states of the world-then clearly no useful information is expected. To have better information, it is necessary for the signal $\tilde{q}$ to be more dispersed. This is because the more dispersed is this signal, the more useful is the information that can be acted on at date $t=\kappa_{1}$. To see this, write the expectations of equations (16) and (17) as

(21)

$$
\begin{aligned}
& E\left[V\left(N_{0}, a_{1}^{*} \mid \tilde{q}\right)\right] \\
& =\left(\frac{D}{r+h_{1}}+\frac{h_{1} h_{2}(C-\delta r)}{r\left(r+h_{1}\right)\left(r+h_{2}\right)}\right) q \\
& \quad+K q^{+}-K \int_{0}^{q^{+}} G(\tilde{q}) d \tilde{q}
\end{aligned}
$$

$$
\begin{aligned}
& E\left[V\left(B_{0}, a_{1}^{*} \mid \tilde{q}\right)\right] \\
& =\frac{C+\delta r+2 \delta h_{1}}{r+h_{1}}+\frac{h_{1} h_{2}(C-\delta r)}{r\left(r+h_{1}\right)\left(r+h_{2}\right)} q \\
& \quad+K q^{-}-K \int_{0}^{q^{-}} G(\tilde{q}) d \tilde{q} .
\end{aligned}
$$

Note that the right-hand expressions in these two equations are decreasing in a meanpreserving spread on the $t=\kappa_{1}$ signal so that $\min \left\{E\left[V\left(N_{0}, a_{1}^{*} \mid \tilde{q}\right)\right], E\left[V\left(B_{0}, a_{1}^{*} \mid \tilde{q}\right)\right]\right\}$ is also decreasing. This conclusion conforms to the well-known result that a more dispersed signal structure is more informative in the sense that it supports a lower expected loss (Blackwell, 1953; Epstein, 1980). That is to say, a meanpreserving spread in the $t=\kappa_{1}$ signals represents an increase in the information structure's informativeness from the viewpoint of $t=0$.

How exactly the increase in informativeness associated with a meaningful resolution of uncertainty affects the initial choice depends on the structure of the distribution of the signal $\tilde{q}$. There are seven conceivable cases. For each case $j \in\{1,2, \ldots, 7\}$, we use Bayes conditioning to develop the threshold level $q_{j}$ above which the initial decision will be to ban.

Case 1. $G\left(\left[0, q^{+}\right)\right)=0$

In this case, the optimal intermediate decision is $a_{1}^{*}=B_{1}$ with probability 1 . Given that, the $t=0$ decision should be to ban if $V\left(N_{0}, B_{1} \mid q\right)>V\left(B_{0}, B_{1} \mid q\right)$, which requires

$$
q>\frac{C+\delta r}{D} \equiv q_{1}
$$

Because $q_{1}<q^{+}$, and given that $q>q^{+}$ with probability 1 , here it is optimal to ban at $t=0$.
Case 2. $G\left(\left[q^{-}, 1\right]\right)=0$

In this case, the optimal intermediate decision is $a_{1}^{*}=N_{1}$ with probability 1 . Given that, the optimal decision at $t=0$ is to not ban if $E\left[V\left(B_{0}, N_{1} \mid \tilde{q}\right)\right]>$ $E\left[V\left(N_{0}, N_{1} \mid \tilde{q}\right)\right]$, which requires

$$
q<\frac{\left(C+\delta r+2 \delta h_{1}\right)}{D} \equiv q_{2}
$$

Because $q<q^{-}$with probability 1 , and $q^{-}<q_{2}$, it is optimal to not ban at $t=0$ in this case.

Case 3. $G\left(\left[q^{-}, q^{+}\right)\right)=0$ and neither Case 1 nor Case 2

Here, the optimal intermediate decision is to ban if $\tilde{q}>q^{+}$and to not ban if $\tilde{q}<q^{-}$. Thus, the regulator's expected $t=0$ losses associated with the two actions are

$$
\begin{aligned}
& E\left[V\left(B_{0}, a_{1}^{*} \mid \tilde{q}\right)\right] \\
& =\int_{q^{+}}^{1} V\left(B_{0}, B_{1} \mid \tilde{q}\right) d G(\tilde{q}) \\
& \quad+\int_{0}^{q^{-}} V\left(B_{0}, N_{1} \mid \tilde{q}\right) d G(\tilde{q}) \\
& E\left[V\left(N_{0}, a_{1}^{*} \mid \tilde{q}\right)\right] \\
& =\int_{q^{+}}^{1} V\left(N_{0}, B_{1} \mid \tilde{q}\right) d G(\tilde{q}) \\
& \quad+\int_{0}^{q^{-}} V\left(N_{0}, N_{1} \mid \tilde{q}\right) d G(\tilde{q}) .
\end{aligned}
$$

The optimal decision at $t=0$ is to ban if $E\left[V\left(N_{0}, a_{1}^{*} \mid \tilde{q}\right)\right]>E\left[V\left(B_{0}, a_{1}^{*} \mid \tilde{q}\right)\right]$, which requires

$$
q>\frac{C+\delta r+2 \delta h_{1} G\left(q^{-}\right)}{D} \equiv q_{3} .
$$

It is readily shown that either decision can occur under this case.

Case 3 may be viewed as a convex combination of the preceding cases. If $G([0$, $\left.\left.q^{-}\right)\right)$is so small as to be negligible, then $\Delta \approx[C+\delta r-q D] /\left(r+h_{1}\right)$. A $t=$ $\kappa_{1}$, reversal of $a_{0}=B_{0}$ is then unlikely and the $t=0$ decision should be similar to that of Case 1. In general, a more dispersed signal at $t=\kappa_{1}$ can increase or decrease the value of $G\left(q^{-}\right)$. This means that if $G(q)$ becomes more dispersed then a $N_{0}$ decision can change to a $B_{0}$ decision or vice versa. 
Case 4. $G\left(\left[q^{-}, q^{+}\right)\right)=1$

Here, the optimal intermediate action is to confirm the initial action $B_{1} \mid B_{0}$ or $N_{1} \mid N_{0}$. So it is optimal to ban if $\int_{0}^{1} V\left(N_{0}, N_{1} \mid \tilde{q}\right) \times$ $d G(\tilde{q})>\int_{0}^{1} V\left(B_{0}, B_{1} \mid \tilde{q}\right) d G(\tilde{q})$, i.e., if

$q>\frac{\left(r+h_{1}+h_{2}\right)(C+\delta r)+2 \delta h_{1} h_{2}}{\left(r+h_{1}+h_{2}\right) D+2 \delta h_{1} h_{2}} \equiv q_{4}$.

Notice that $q_{4} \equiv \hat{q}$ as defined in relation (10), and the reason is straightforward. In Case 4, the capacity to adapt the regulation to intermediate-stage information is of no relevance because, given the distribution of $\tilde{q}$ that characterizes this case, it will never happen. As in Case 3, choices of cost parameters can support both $t=0$ decisions. To see this, set $r=0$ and $h_{1}=$ $h_{2}=1$ so that the criterion becomes $q>$ $(C+\delta) /(D+\delta)$.

Case 5. $G\left(\left[0, q^{-}\right)\right)=0, G\left(\left[q^{-}, q^{+}\right)\right) \neq 0$, and $G\left(\left[q^{+}, 1\right]\right) \neq 0$

In this case, the optimal intermediate policy is to ban if $\tilde{q}>q^{+}$and to stick with the original decision if $\tilde{q} \leq q^{+}$. Thus, the optimal decision at $t=0$ is to ban if $E\left[V\left(N_{0}, a_{1}^{*} \mid \tilde{q}\right)\right]>E\left[V\left(B_{0}, a_{1}^{*} \mid \tilde{q}\right)\right]$, where

$$
\begin{aligned}
& E\left[V\left(N_{0}, a_{1}^{*} \mid \tilde{q}\right)\right] \\
& =\int_{q^{-}}^{q^{+}} V\left(N_{0}, N_{1} \mid \tilde{q}\right) d G(\tilde{q}) \\
& \quad+\int_{q^{+}}^{1} V\left(N_{0}, B_{1} \mid \tilde{q}\right) d G(\tilde{q}) \\
& E\left[V\left(B_{0}, a_{1}^{*} \mid \tilde{q}\right)\right] \\
& =\int_{q^{-}}^{q^{+}} V\left(B_{0}, B_{1} \mid \tilde{q}\right) d G(\tilde{q}) \\
& \quad+\int_{q^{+}}^{1} V\left(B_{0}, B_{1} \mid \tilde{q}\right) d G(\tilde{q}) .
\end{aligned}
$$

No clean analog to (22), (23), and (27) exists. Condition $E\left[V\left(N_{0}, a_{1}^{*} \mid \tilde{q}\right)\right]=$ $E\left[V\left(B_{0}, a_{1}^{*} \mid \tilde{q}\right)\right]$ implicitly defines the $t=$ 0 level of $q$, call it $q_{5}$, above which banning is optimal.

As in Case 3, even conditional on the case, the particulars of information structure $\{\tilde{q}, G(\tilde{q})\}$ determine the optimal $t=0$ decision. Observe that, for the domain of
Case 5 , the function $A\left(\tilde{q} ; q^{+}, q^{-}\right)$is convex in $\tilde{q}$ (cfr. figure 5) so that a meanpreserving spread in information structure increases $\Delta$. Numerical examples readily show, assuming the case conditions continue to hold, that a more informative $\{\tilde{q} ; G(\tilde{q})\}$ can change the $t=0$ decision from $B_{0}$ to $N_{0}$, but convexity ensures that the reverse cannot occur.

Case 6. $G\left(\left[0, q^{-}\right)\right) \neq 0, G\left(\left[q^{-}, q^{+}\right)\right) \neq 0$, and $G\left(\left[q^{+}, 1\right]\right)=0$

In this case, the optimal intermediate policy is to not ban if $\tilde{q} \leq q^{-}$, and to stick with the original decision under all other signals. Thus, the optimal decision at $t=0$ is to ban if $E\left[V\left(N_{0}, a_{1}^{*} \mid \tilde{q}\right)\right]>E\left[V\left(B_{0}, a_{1}^{*} \mid \tilde{q}\right)\right]$ where

$$
\begin{aligned}
& E\left[V\left(N_{0}, a_{1}^{*} \mid \tilde{q}\right)\right] \\
& =\int_{0}^{q^{-}} V\left(N_{0}, N_{1} \mid \tilde{q}\right) d G(\tilde{q}) \\
& \quad+\int_{q^{-}}^{q^{+}} V\left(N_{0}, N_{1} \mid \tilde{q}\right) d G(\tilde{q}) \\
& E\left[V\left(B_{0}, a_{1}^{*} \mid \tilde{q}\right)\right] \\
& =\int_{0}^{q^{-}} V\left(B_{0}, N_{1} \mid \tilde{q}\right) d G(\tilde{q}) \\
& \quad+\int_{q^{-}}^{q^{+}} V\left(B_{0}, B_{1} \mid \tilde{q}\right) d G(\tilde{q}) .
\end{aligned}
$$

As in Case 5, no clean analog of (22), (23), and (27) exists.

In figure 5 and for the domain of Case $6, \Delta$ is concave so that a mean-preserving spread in information structure decreases $\Delta$. Again, and assuming the case conditions continue to hold, numerical examples show that a more informative $\{\tilde{q} ; G(\tilde{q})\}$ can change the $t=0$ decision from $N_{0}$ to $B_{0}$. But concavity ensures a $B_{0}$ will not be overturned to $N_{0}$ in the presence of more information.

Case 7. $G\left(q^{-}\right) \neq 0, G\left(\left[q^{-}, q^{+}\right)\right) \neq 0$, and $G\left(\left[q^{+}, 1\right]\right) \neq 0$

The absence of uniform curvature on the function $A\left(\tilde{q} ; q^{+}, q^{-}\right)$means that a more informative signal structure can lead to an overturn of decision $N_{0}$ to $B_{0}$ or an overturn of decision $B_{0}$ to $N_{0}$.

RESULT 4. Signal dispersion and flexibility do notmatter for Cases 1, 2, and 4. In such cases, the 
informativeness of the signal is inconsequential for the optimal decision at $t=0$. For the other cases, signal dispersion coupled with flexibility leads to lower expected costs and can result in the initial ban decision to be changed to a no ban decision (Cases 3, 5, and 7) and in an initial no ban decision to be changed to a ban decision (Cases 3, 6, and 7).

These conclusions of our model are related to some fundamental results in the economics of information. Particularly germane is Theorem 1 in Epstein, which highlights the crucial role played by the curvature (in the distribution of uncertainty) of the marginal condition of the initial problem. In our case, the critical element concerns the curvature of the function $A\left(\tilde{q} ; q^{+}, q^{-}\right)$.

A notable feature of Result 4 is that the recognition that better quality information is coming does not necessarily favor the status quo. ${ }^{17}$ This is due to the fact that the function $A\left(\tilde{q} ; q^{+}, q^{-}\right)$does not have a uniform curvature on the entire domain. Specifically, as discussed earlier and as illustrated in figure 5 , the function in question is concave on $\left[0, q^{+}\right]$and convex on $\left[q^{-}, 1\right]$. An increase in the dispersion of the signal-i.e., more information-has opposite effects on the expected value of the function $A\left(\tilde{q} ; q^{+}, q^{-}\right)$, and thus has opposite impacts on the decision problem, depending on whether the function in question is concave or convex. When the signal's distribution spans both concave and convex portions (as in cases 3 and 7) then ambiguity arises. Interestingly, again, such a situation allows for the (perhaps counterintuitive) result that a more informative signal may actually increase the propensity to ban at the initial stage (i.e., it may move the initial decision away from the status quo). As will be explained in the next section, this ambiguity concerning the effect on decisions is not shared with the effect of more earlystage research on decisions. Both decrease the expected loss to the regulator, but the effect on decisions is clearer for more early-stage research.

\section{Effect of More Early-Stage Research}

The arrival of new scientific information can be affected by scientific research, and indeed it is common to see calls for bans and regula-

\footnotetext{
${ }^{17}$ We reiterate that the conclusions of Result 4 are obviously predicated on the assumption that the conditions defining each case do not change as the information structure is changed.
}

tion coupled with calls for more investment in research on the problem at hand. How compelling are such calls in the context of our model? To address this question, note that in our model the arrival of information is governed by the parameters of the exponential distributions $\left(h, h_{1}\right.$ and $\left.h_{2}\right)$. Consider first the initial model without intermediate flexibility, leading to the decision rule of equation (5). Here the mean time to arrival of intermediate information is $1 / h$, and one can think of investment in scientific research as increasing the parameter $h$. The right-hand side of equation (5) immediately yields $\partial q_{\delta, h} / \partial h>0$. Thus, investments that speed up the expected resolution of uncertainty have the collateral effect of tilting the optimal initial decision further towards the status quo. Indeed, because $\lim _{h \rightarrow \infty} q_{\delta, h}=$ 1 , one would always want to wait if news was likely to come very soon.

A similar conclusion can be obtained when intermediate flexibility is allowed. Specifically, suppose that an investment in more scientific research is interpreted as increasing parameter $h_{1}$ (i.e., reducing the expected time to arrival of the intermediate information). To analyze how such an increase in $h_{1}$ will affect the optimal decision, write $\Delta$ in (18) as

$$
\begin{aligned}
\Delta & =\frac{C+\delta r-q D+2 \delta h_{1}(1-\phi)}{r+h_{1}} \\
\phi & =-\frac{\left(r+h_{1}\right)}{2 \delta h_{1}} E\left[A\left(\tilde{q} ; q^{+}, q^{-}\right)\right] .
\end{aligned}
$$

Denominator $r+h_{1}$ is always positive. Furthermore, it can be shown that $\phi \in[0$, 1] because of the bounds on the function $A\left(\tilde{q} ; q^{+}, q^{-}\right)$(cfr. figure 5). Consequently, if $\Delta>0$, then an increase in the value of $h_{1}$ cannot change the sign of $\Delta$. Hence, when the regulator's initial decision is to not ban, this decision cannot be affected by more research leading to a shortening of $E\left[\kappa_{1}\right]$. On the other hand, when $\Delta<0$, an increase in the value of $h_{1}$ could in fact change the sign of $\Delta$. Thus, if the candidate's $t=0$ decision is to ban, a commitment to more research leading to an increase in $h_{1}$ could overturn the optimal initial decision.

RESULT 5. Research funding and a regulatory ban can be viewed as "substitutes." A commitment to undertake more scientific research leading to earlier resolution of uncertainty tilts the 
optimal initial decision further towards the status quo.

\section{How Much Scientific Research?}

Although governments often separate research funding decision-making from rulemaking, the foregoing suggests that a coordinated response to a regulation problem in the absence of sufficient scientific information should involve both the regulation choice itself and the extent of funding for research that improves the information of the regulator. In this section we investigate briefly the interaction between research decisions and regulatory decisions. To keep the analysis tractable, we limit attention to the initial framework without intermediate flexibility. The modeling approach is to let $h \equiv h_{0}+\widehat{h}$, where $h_{0}$ is the baseline parameter governing the resolution of uncertainty (which depends on research that will anyway be undertaken in the economy), and $\widehat{h} \geq 0$ is the addition to this parameter due to directed research under the control of the government. Thus, the baseline exogenous expected arrival of information is $1 / h_{0}$, and the expected arrival date of information when additional research is undertaken is $1 /\left(h_{0}+\widehat{h}\right)$ (so that the regulator can accelerate the resolution of uncertainty by funding additional research).

The cost of the additional research effort is parameterized as $\rho \widehat{h}$, where $\rho>0$ is the unit cost (which reflects many things, including the state of knowledge in a discipline and the particular research effort that is necessary). By using (3) and (4) when $h \equiv h_{0}+\widehat{h}$, the regulator's loss minimization problems, conditional on a given $\{B, N\}$ policy choice, are:

$$
\begin{aligned}
L(B)= & \min _{\widehat{h} \geq 0} \delta_{1}+\frac{C+\delta_{2}\left(h_{0}+\widehat{h}\right)}{r+h_{0}+\widehat{h}} \\
& +\frac{q\left(h_{0}+\widehat{h}\right)\left(C-\delta_{2} r\right)}{r\left(r+h_{0}+\widehat{h}\right)}+\rho \widehat{h}
\end{aligned}
$$

$L(N)$

$$
=\min _{\widehat{h} \geq 0} q\left(\frac{C\left(h_{0}+\widehat{h}\right)+D r+\delta_{1}\left(h_{0}+\widehat{h}\right) r}{r\left(r+h_{0}+\widehat{h}\right)}\right)+\rho \widehat{h} .
$$

The first-order conditions for an interior solution are $(1-q)\left(C-\delta_{2} r\right) /\left(r+h_{0}+\widehat{h}\right)^{2}=$ $\rho$ under ban and $q\left(D-C-\delta_{1} r\right) /\left(r+h_{0}+\right.$ $h)^{2}=\rho$ under no ban. In each case, secondorder conditions for convexity are satisfied so that optimal solutions $\widehat{h}_{B}^{*}$ and $\widehat{h}_{N}^{*}$, respectively, attain the unique values

$$
\widehat{h}_{B}^{*}=\max \left\{\left(-r-h_{0}+\sqrt{(1-q)\left(C-\delta_{2} r\right) / \rho}\right), 0\right\}
$$

$$
\widehat{h}_{N}^{*}=\max \left\{\left(-r-h_{0}+\sqrt{q\left(D-C-\delta_{1} r\right) / \rho}\right), 0\right\} .
$$

Upon defining the critical points:

$$
\begin{aligned}
& q^{B} \equiv 1-\left(r+h_{0}\right)^{2} \rho /\left(C-\delta_{2} r\right) \\
& q^{N} \equiv\left(r+h_{0}\right)^{2} \rho /\left(D-C-\delta_{1} r\right),
\end{aligned}
$$

from equations (35) and (36) the following four cases arise regarding whether it is necessary to engage in research efforts beyond the exogenous level underlying $h_{0}$.

Case (i). $q \geq q^{B}$ and $q \leq q^{N}$. In this case, additional research is not warranted regardless of the initial ban decision taken. The case was analyzed previously, with decision rule given in (5) where $h=h_{0}$. This case will apply when the values of $h_{0}$ and $\rho$ are sufficiently high.

Case (ii). $q \geq q^{B}$ and $q>q^{N}$. In this case, additional research is warranted only when the initial decision is not to ban. From (37) and (38), it is apparent that the conditions of this case will be true when $\left(r+h_{0}\right)^{2} \rho$ is large, $D-C-\delta_{1} r$ is larger by a sufficient magnitude, and $q$ is sufficiently small. Even though the probability of harm is low, the loss if harm does occur is sufficient to elicit additional research when the decision is not to ban.

Case (iii). $q<q^{B}$ and $q \leq q^{N}$. In this case, additional research is warranted only when the initial decision is to ban. From (37) and (38), it is apparent that the conditions of this case will be true when $\left(r+h_{0}\right)^{2} \rho$ is large, $C-\delta_{2} r$ is larger by a sufficient magnitude, and $q$ has value close to 1 . Even though the probability of harm is high, sector losses under a ban are sufficient to elicit additional research were a ban to be put in place.

Case (iv). $q<q^{B}$ and $q>q^{N}$. This is the most interesting case and arguably the most plausible for situations involving pressing scientific problems, and thus we will consider this case in more detail in the remainder of the section. It occurs when $r+h_{0}$ 
and $\rho$ are sufficiently low in value. In this case, additional research is warranted regardless of the initial ban decision taken. By using the optimal solutions in (35) and (36), the value functions of the minimization problems in (33) and (34) are

$$
\begin{aligned}
& L(B)=\delta_{1}+\delta_{2}(1-q)+\frac{q C}{r}-r \rho \\
& -h_{0} \rho+2 \sqrt{\rho(1-q)\left(C-\delta_{2} r\right)} \\
& L(N)=\frac{q\left(C+\delta_{1} r\right)}{r}-r \rho-h_{0} \rho \\
& +2 \sqrt{\rho q\left(D-C-\delta_{1} r\right)} .
\end{aligned}
$$

Thus, the decision is to ban if and only if $L(N) \geq L(B)$, which requires:

$$
\hat{h}_{N}^{*}-\widehat{h}_{B}^{*} \geq \frac{\left(\delta_{1}+\delta_{2}\right)(1-q)}{2 \rho} .
$$

Observe that if $q=0$ then (41) is never true. If $q=1$, then it is true given that $D>$ $C+\delta_{1} r$. If the left-hand side of (41) has value 0 then $q=\left(C-\delta_{2} r\right) /\left(D-\delta_{1} r-\delta_{2} r\right)$ and the right-hand side of (41) is strictly positive at this $q$ value (again, given $D>$ $\left.C+\delta_{1} r\right)$. In addition, the left-hand side of (41) is increasing in $q$ while the righthand side is decreasing in $q$, so continuity ensures that there is a unique value of $q$, label it $q^{R}$ (where superscript $R=$ research), such that (41) holds with equality. From Result 5 we know that the "no ban" region increases as $h$ increases, and hence $q^{R}>q_{\delta, h_{0}}$. Furthermore, $\widehat{h}_{N}^{*}>\widehat{h}_{B}^{*}$ when both expressions in (35) and (36) are evaluated at $q=q^{R}$.

RESUlt 6. Consider the case with no intermediate flexibility, where $\left(r+h_{0}\right)^{2} \rho$ is sufficiently low in value and it is possible to speed up the resolution of uncertainty by additional scientific research. Then there exists a value $q^{R} \in(0,1)$ such that it optimal not to ban if $q \leq q^{R}$ and it is optimal to ban if $q>q^{R}$. The optimal level of research increases with $q$ on $q \leq q^{R}$ and decreases on $q>q^{R}$, i.e., $d \widehat{h}_{N}^{*} / d q \geq 0$ on $q \leq q^{R}$ and $d \widehat{h}_{B}^{*} / d q \leq 0$ on $q>q^{R}$. In addition, when $q=q^{R}$ then optimal research under no ban is larger than under a ban.

The intuition for this analysis is as follows. At low and high $q$ values the $t=0$ regulation is unlikely to be overturned when the truth is finally known. There is not a strong incentive to accelerate the expected time of acquiring additional information. When the value of $q$ is more central, then the risk of having had the wrong policy in place at $t=0$ is larger and so more research should be undertaken. This need for more research is particularly acute when no ban is put in place at $t=0$ because, as result 5 has already indicated, the decision to ban substitutes for research.

\section{Conclusion}

Regulatory activism that limits the use of efficient technologies otherwise favored by producers, or limits the adoption of new technologies, has at least two contrasting effects. On the one hand new regulation has an expected (gross) benefit because it limits exposure to potential risks, and on the other it introduces an expected cost because it promotes the use of less efficient technologies. Striking an optimal trade-off between costs and benefits is difficult because decisions are typically required under imperfect scientific information.

In such a setting some would argue for a cautious or "precautionary" approach to regulation on the grounds that restrictive regulation could anyway be abandoned in the future, if the resolution of scientific uncertainty were to warrant that. But drastic changes in production or distribution patterns forced by regulation also entails adjustment costs that are sunk, i.e., such costs cannot be recovered even if the regulation in question is modified at a future date. In this paper, we have argued that such sunk costs must be accounted for in the computation of the costs and benefits trade-offs of regulation. More importantly, the existence of such sunk costs changes the inherent structure of the regulation problem once it is realized that scientific uncertainty is resolved gradually over time.

Our objective here has been to clarify how adjustment costs affect the dynamics of regulatory decisions as scientific information becomes more complete. Broadly speaking, the existence of adjustment costs creates inertia and favors the status quo. But adjustment costs also change how the optimal regulation responds to better scientific information. The knowledge that more information is on the way can increase or decrease the incentive to put an early ban in place. We show how parameters other than the cost per unit time to producers and the magnitude of contingent damage per unit time matter. Other critical features in the model are the extent of adjustment costs 
that might be incurred, the extent of information that will be revealed, the structure of that information, the stochastic attributes of the information revelation date, and the unit cost of research. But, regardless of the information structure, a regulator may sometimes be able to decide early and with high confidence if reliable estimates of $C$ and $D$ can be obtained.

Where one starts in the process does matter as well. If a technology is banned before adoption can occur, as was the case with GM crops in many European countries, then the existence of adjustment costs in the presence of imperfect information should act as a deterrent to lifting the ban. Given these qualifications, though, there is no a priori reason to believe that a precautionary ban is the best principle when seeking to manage BSE or any other crisis for which current science provides less-than-conclusive guidance.

\section{[Received May 2004; accepted June 2005.]}

\section{References}

Arrow, K.J., and A.C. Fisher. 1974. "Environmental Preservation, Uncertainty, and Irreversibility." Quarterly Journal of Economics 88:31219.

Blackwell, D. 1953. "Equivalent Comparisons of Experiments." Annals of Mathematical Statistics 24:265-73.

Bradley, R. 2003. "BSE Risks for Humans Consuming Beef and Beef Products: How Any Risks Are Managed." Veterinary Research Communications 27:15-23.

Commission of the European Communities. 2000. "Communication from the Commission on the Precautionary Principle." $\operatorname{COM(2000)} 1$ final, Brussels, February.

—. 2001. "Commission Staff Working Paper on the Processing, Disposal and Uses of Animal By-Product in Member States." SEC(2001) 1889, Brussels, November.

—. 2003. "European Legislative Framework for GMOs Is Now in Place." Press Release, IP/03/1056, Brussels. 22 July.

Dixit, A., and R.S. Pindyck. 1994. Investment under Uncertainty. Princeton NJ: Princeton University Press.

Eeckhoudt, L., C. Gollier, and N. Treich. 2005. “Optimal Consumption and the Timing of the Resolution of Uncertainty." European Economic Review 49:761-73.
Epstein, L.G. 1980. "Decision Making and the Temporal Resolution of Uncertainty." International Economic Review 21:269-83.

Fisher, A.C., and U. Narain. 2003. "Global Warming, Endogenous Risk, and Irreversibility." Environmental and Resource Economics 25:395416.

Freestone, D., and E. Hey, eds. 1996. The Precautionary Principle and International Law. The Hague: Kluwer.

Gollier, C. 2001. "Should We Beware the Precautionary Principle?" Economic Policy 16:30221.

Gollier, C., B. Jullien, and N. Treich. 2000. "Scientific Progress and Irreversibility: An Economic Interpretation of the 'Precautionary Principle." Journal of Public Economics 75:22953.

Hanemann, W.M. 1989. "Information and the Concept of Option Value." Journal of Environmental Economics and Management 16:23-37.

Henry, C. 1974. "Investment Decisions Under Uncertainty: The 'Irreversibility Effect." American Economic Review 64:1006-12.

Hill, A.F., S. Joiner, J. Linehan, M. Desbruslais, P.L. Lantos, and J. Collinge. 2000. "SpeciesBarrier-Independent Prion Replication in Apparently Resistant Species." Proceedings of the National Academy of Sciences of the United States 97:10248-253.

Jones, R.A., and J.M. Ostroy. 1984. "Flexibility and Uncertainty." Review of Economic Studies 51:13-32.

Kolstad, C.D. 1996a. "Fundamental Irreversibilities in Stock Externalities." Journal of Public Economics 60:221-33.

- 1996b. "Learning and Stock Effects in Environmental Regulations: The Case of Greenhouse Gas Emissions." Journal of Environmental Economics and Management 31:1-18.

Kreps, D.M., and E.L. Porteus. 1978. "Temporal Resolution of Uncertainty and Dynamic Choice Theory." Econometrica 46:185200.

Loury, G.C. 1979. "Market Structure and Innovation." Quarterly Journal of Economics 93:395410.

Mossin, J. 1969. “A Note on Uncertainty and Preferences in a Temporal Context." American Economic Review 59:172-74.

Official Journal of the European Communities. 2002. Regulation (EC) No 1774/2002 of the European Parliament and of the Council. L 273, Brussels, 3 October.

Pindyck, R.S. 2002. "Optimal Timing Problems in Environmental Economics." Journal of Economic Dynamics and Control 26:1677-97. 
Raine, M. 2003. "Waste Disposal Becomes Costly." Western Producer, December 17, http:// www.producer.com/articles/20031211/special_ report/20031211rendering01.html.

Schuff, S. 2004a. "Policy Changes to Protect Public, Animal Well-Being." Feedstuffs, January 26, p. 1 and p. 4.

- 2004b. "BSE Surveillance Key Theme During Committee Hearing." Feedstuffs, January 12, p. 6 and p. 41.

Smith, P.G. 2003. "The Epidemics of Bovine Spongiform Encephalopathy and Variant CreutzfeldtJakob Disease: Current Status and Future Prospects." Bulletin of the World Health Organization 81:123-30.

Spence, M., and R. Zeckhauser. 1972. "The Effect of Timing of Consumption Decisions and the Resolution of Lotteries on the Choice of Lotteries." Econometrica 40:401-03.
Taylor, H.M., and S. Karlin. 1984. An Introduction to Stochastic Modeling. Orlando, FL: Academic Press.

Ulph, A., and D. Ulph. 1997. "Global Warming, Irreversibility and Learning." Economic Journal 107:636-50.

U.S. Food and Drug Administration. 2002. Guidance for Industry: Revised Preventive Measures to Reduce the Possible Risk of Transmission of Creutzfeldt-Jakob Disease (CJD) and Variant Creutzfeldt-Jakob Disease (vCJD) by Blood and Blood Products. Center for Biologics Evaluation and Research, January.

Will, R.G., J.W. Ironside, M. Zeidler, S.N. Cousens, K. Estibeiro, A. Alperovitch, S. Poser, M. Pocchiari, A. Hofman, and P.G. Smith. 1996. "A New Variant of Creutzfeldt-Jakob Disease in the UK." Lancet 347:921-25. 